\title{
AVALIAÇÃO DAS INTERAÇÕES MEDICAMENTOSAS POTENCIAIS EM PRESCRIÇÕES DE PACIENTES EM UNIDADE DE TERAPIA INTENSIVA
}

\section{Cristiane Carla Dressler Garske}

Graduada no curso de Farmácia pela Universidade de Santa Cruz do Sul (UNISC), Brasil.

E-mail: crisdressler2005@yahoo.com.br

\section{Betina Brixner}

Farmacêutica; Especialista em análises clínicas e toxicológicas pela Universidade de Santa Cruz do Sul (UNISC), Brasil.

\section{Alice Pereira Freitas}

Graduada no curso de Farmácia pela Universidade de Santa Cruz do Sul (UNISC), Brasil.

\section{Ana Paula Helfer Schneider}

Farmacêutica; Mestre pelo Programa de Pós-Graduação em Saúde Coletiva na Universidade do Vale do Rio dos Sinos (UNISINOS), Brasil.
RESUMO: A identificação e prevenção de interações entre medicamentos estão diretamente relacionadas com a segurança do paciente e são de grande importância no ambiente hospitalar. O objetivo deste trabalho foi identificar e avaliar a existência de interações medicamentosas potenciais na farmacoterapia prescrita. Trata-se de estudo observacional, com 308 prescrições de 195 pacientes internados na Unidade de Terapia Intensiva de um hospital de ensino, realizado de agosto a dezembro de 2015. Para identificação das interações utilizouse a base de dados Micromedex ${ }^{\circledR}$. Das 308 prescrições analisadas, 101 apresentaram pelo menos uma interação medicamentosa, totalizando 173 interações, referentes a 71 pacientes. Destes, 43 eram do sexo masculino, com idade média de 61,9 anos. A média de internação na unidade foi de 7 dias. Nas 101 prescrições que apresentaram interações medicamentosas, foi prescrito o total de 1.349 medicamentos com média de 13,3 medicamentos/paciente/dia. Identificar essas interações possibilita evitar situações de insucesso terapêutico e toxicidade medicamentosa.

PALAVRAS-CHAVE: Cuidados críticos; Prescrições de medicamentos; Interações de medicamentos.

\section{EVALUATION OF POTENTIAL DRUG INTERACTIONS IN PRESCRIPTIONS FOR PATIENTS IN INTENSIVE CARE UNITS}

ABSTRACT: The identification and the interaction of drugs are directly related to patient's safety and are highly relevant within the hospital. Current analysis identifies and evaluates potential drugs interactions within prescribed pharmacotherapy. The study, undertaken between August and December 2015, comprises 308 prescriptions of 195 patients hospitalized in the UTI of a university hospital. Micromedex ${ }^{\circledR}$ database identified interactions. Further, 101 out of 308 prescriptions revealed at least one drug interaction, with a total of 173 interactions with 71 patients, of which 43 were males with an average age of 61,9 years. Mean hospitalization period in the ICUs was 7 days. The 101 prescriptions with drug interactions totaled 1,349 drugs, at an average of 13,3 drugs/patient/day. The identification of these interactions avoids therapeutic failure and toxicity.

KEY WORDS: Critical care; Drug prescriptions; Drug interactions. 


\section{INTRODUÇÃO}

A Unidade de Terapia Intensiva (UTI) é um centro de monitoramento contínuo de pacientes em estado grave, com descompensação de um ou mais sistemas orgânicos, onde há possibilidade de restabelecer a homeostase, por meio de suporte altamente tecnológico e tratamento intensivo por equipe multiprofissional especializada (RODRIGUES; OLIVEIRA, 2010). Devido às graves condições clínicas que os pacientes internados na UTI se encontram, acabam fazendo uso de um elevado número de medicamentos, visando à cura $\mathrm{e}$ a oferta de suporte farmacoterapêutico. Prescrever e administrar medicamentos exige conhecimento científico e habilidade técnica, para garantir a segurança na terapêutica medicamentosa administrada. Devido à polifarmacoterapia, os pacientes internados em UTI, são mais propensos ao risco de interações medicamentosas (RODRIGUES; OLIVEIRA, 2010; PAPADOPOULOS; SMITHBURGER, 2010; SMITHBURGER; KANEGILL; SEYBERT, 2010; SECOLI, 2010; SPRIET et al., 2009).

$\mathrm{O}$ acesso à assistência médica e aos medicamentos, não é, necessariamente, sinônimo de melhores condições de saúde ou qualidade de vida, pois os maus hábitos de prescrições, as falhas na dispensação e a automedicação inadequada podem levar a tratamentos ineficazes e pouco seguros. É evidente que a possibilidade de receber o tratamento adequado, conforme e quando necessário, reduz a incidência de agravos à saúde, bem como a mortalidade para muitas doenças. Muitos dos problemas relacionados aos medicamentos são causados por interações medicamentosas (DOURADO; BARRETO; COELHO, 2007).

Interação medicamentosa é aquela quando um medicamento é alterado pela ação de outro medicamento, fitoterápico, alimento, bebida ou algum agente químico ambiental e contribui significativamente para maiores incidências de reações adversas no âmbito hospitalar, ocasionando uma maior permanência do paciente no hospital e aumento de custos com a saúde (BRASIL, 2010).

Os efeitos resultantes das interações medicamentosas podem ser benéficos, quando aumentam a eficácia da medicação ou reduzem efeitos adversos, mas também podem ser nocivos, diminuindo a eficácia do medicamento e/ou aumento da toxicidade, dependendo dos fatores ligados ao medicamento, ao paciente ou as condições de utilização dos medicamentos (HAMMES et al., 2008; OGA, 2008). Estando relacionada com a segurança do paciente, a detecção e prevenção das interações medicamentosas são de grande importância no ambiente hospitalar, diminuindo causas de morbimortalidade nos pacientes internados e, consequentemente, reduzindo impacto no âmbito clínico e econômico (SMITHBURGER; KANE-GILL; SEYBERT, 2010; SPRIET et al., 2009). Diante do exposto, o presente estudo tem como objetivo identificar e avaliar a existência de interações medicamentosas potenciais entre medicamentos prescritos na UTI de um hospital de ensino.

\section{METODOLOGIA}

Estudo retrospectivo de delineamento observacional. A população alvo deste estudo foi constituída de pacientes internados na UTI de um hospital de ensino do interior do estado do Rio Grande do Sul, Brasil, no período de agosto a dezembro de 2015. Foram analisadas 309 prescrições. O levantamento de dados foi através dos prontuários dos pacientes e análise das prescrições.

Como critério de inclusão considerou-se todas as prescrições médicas dos pacientes que internaram na UTI adulto no período e com idade superior a 18 anos. As informações coletadas foram Código Internacional da Doença (CID), sexo, idade, tempo de internação na unidade e número de medicamentos prescritos/paciente/ dia, interações medicamentosas potenciais encontradas.

A busca e a classificação das possíveis interações medicamentosas nas prescrições foram realizadas com auxílio da base de dados Micromedex ${ }^{\circledR}$ (Micromedex ${ }^{\circledR}$ Healthcare Series), classificando-as como: contraindicada (quando o uso concomitante dos medicamentos não é indicado) e; maior (quando a interação apresenta ameaça para a vida do paciente e/ou exige intervenção médica para reduzir ou prevenir graves efeitos adversos). Foram 
consideradas somente as interações medicamentosas que apresentaram evidência científica classificada como excelente (quando estudos controlados estabeleceram de modo claro a existência da interação) e boa (quando a documentação sugere com veemência a existência da interação, mas faltam estudos controlados realizados de modo adequado). Foram excluídas as interações medicamentosas classificadas como moderadas ou menores e aquelas que apresentavam documentação razoável ou desconhecida. Após a coleta dos dados, a análise estatística foi realizada através do software Microsoft Excel 2007, registrando os dados originais e para a análise exploratória, posteriormente gerando quadros e gráficos.

Este estudo foi isento de termo de consentimento livre e esclarecido, devido ao anonimato dos pacientes e faz parte de um estudo maior intitulado "Ocorrência de interações e incompatibilidades medicamentosas e intervenções farmacêuticas em unidades de internação hospitalar em hospital de ensino no interior do Rio Grande do Sul" o qual foi submetido e aprovado pelo Comitê de Ética em Pesquisa (CEP) da Universidade de Santa Cruz do Sul (UNISC) no dia 30/09/2015, sob o parecer $n^{0} 1.252 .586 / 15$.

\section{RESULTADO}

O presente estudo avaliou 308 prescrições médicas de 195 pacientes internados na UTI. Das prescrições analisadas, 101 (32,8\%) apresentaram pelo menos uma interação medicamentosa e eram referentes a 71 pacientes internados. Nestes 71 pacientes, foram encontrados 48 CID diferentes; os mais prevalentes foram o Z000 (Exame Geral) em 5 pacientes, seguido do CID J441 (Doença Pulmonar Obstrutiva Crônica) também em 5 pacientes e CID I743 (Embolia e trombose de artérias dos membros inferiores) e CID I200 (Angina Instável) em 4 pacientes.Dos 71 pacientes que apresentaram alguma interação medicamentosa na prescrição, $28(39,4 \%)$ eram do sexo feminino e $43(60,6 \%)$ do sexo masculino, a idade média entre eles foi de 61,9 anos, com idade mínima e máxima de 18 a 89 anos, respectivamente. Observou-se que $64,8 \%$ dos pacientes eram idosos, com idade $\geq 60$ anos.

Nas 101 prescrições que apresentaram interações medicamentosas, foram prescritos um total de 1.349 medicamentos. O máximo de medicamentos prescritos encontrados por pacientes nessas prescrições diárias foram 21 medicamentos e o mínimo foram de 6 medicamentos, sendo a média de 13,3 medicamentos prescritos/paciente/dia.

Em relação às interações medicamentosas, foram encontradas 173 interações entre medicamentos, sendo que uma dessas foi classificada com gravidade contraindicada e, 172 interações com gravidade maior. Quanto à evidência científica, 31 foram consideradas excelentes e 142 classificadas como boa.

As interações mais frequentes encontradas neste estudo estão descritas do Quadro 1. O medicamento mais envolvido em interação medicamentosa foi a morfina (34), seguido de diazepam (30), ácido acetilsalicílico (25), 
Quadro 1. Interações entre medicamentosos mais encontrados nas análises das prescrições de pacientes internados na UTI de um hospital de ensino do interior do estado do Rio Grande do Sul - RS, no ano de 2015

\begin{tabular}{|l|l|l|l|}
\hline INTERAÇÃo & SEVERIDADE & RESULTADO & $\begin{array}{l}\text { FREQUÊNCIA } \\
\text { (\%) }\end{array}$ \\
\hline Ácido acetilsalicílico + Enoxaparina & Maior & $\begin{array}{l}\text { Quando administrados concomitantemente, pode } \\
\text { aumentar o risco de eventos hemorrágicos. Monitorar } \\
\text { sinais e sintomas do paciente }\end{array}$ & 10,4 \\
\hline Diazepam + Morfina & Maior & $\begin{array}{l}\text { O uso concomitante pode resultar em depressão } \\
\text { respiratória quando esses medicamentos são utilizados em } \\
\text { combinação. A redução da dose de um outro ou ambos } \\
\text { fármacos pode ser necessário }\end{array}$ & 9,2 \\
\hline Diazepam + Fenitolina & Maior & $\begin{array}{l}\text { O uso concomitante do diazepam e fenitolina pode } \\
\text { resultar em alteraçóes nas concentraçóes séricas da } \\
\text { fenitolina }\end{array}$ & 6,9 \\
\hline Midazolam + Morfina & Maior & $\begin{array}{l}\text { O uso concomitante pode resultar em depressão } \\
\text { respiratória. Monitor para a depressão respiratória quando } \\
\text { esses medicamentos são utilizados em combinação. A } \\
\text { redução da dose de um ou ambos os fármacos pode ser } \\
\text { necessário. }\end{array}$ & 5,2 \\
\hline Enalapril + Espironolactona & Maior & $\begin{array}{l}\text { O uso concomitante destes medicamentos pode resultar } \\
\text { em hipercalemia. Monitorar os níveis de potássio sérico. }\end{array}$ & 4,6 \\
\hline
\end{tabular}

fenitoína (25) e enoxaparina (19). Além disso, a média de dias que estes pacientes permaneceram internados na UTI foi 7 dias, variando entre um dia até 61 dias.

\section{DISCUSSÃO}

O perfil de um paciente admitido em UTI juntamente com a propensão de um elevado número de medicamentos prescritos a este, são fatores que podem explicar o aumento da incidência de interações medicamentosas quando comparado com outras unidades hospitalares (ALMEIDA; AKAMINE, 2007).

Neste estudo, o principal CID encontrado nos pacientes que apresentaram interação entre medicamentos foi o Z000, referente ao exame médico geral, seguido do CID J441 (Doença Pulmonar Obstrutiva Crônica), CID I743 (Embolia e trombose de artérias dos membros inferiores) e CID I200 (Angina Instável). Com resultados semelhantes ao encontrado neste estudo, um hospital no interior da Bahia apresentou como principais CIDs, nas prescrições contendo interações medicamento-medicamento, aqueles classificados na categoria S (lesão e outras consequências de causas externas), categoria J (seguidas de doenças do aparelho respiratório) e categoria I (circulatório) (GONÇALVES et al., 2016). Apesar dos resultados semelhantes, sabe-se que a classificação por CIDs tem em vista a padronização e classificação de doenças, a qual é muito particular do perfil dos pacientes atendidos nos hospitais, levando em consideração as referências de atendimentos prestados por essas instituições.

O monitoramento das prescrições de UTI é muito importante diante da grande quantidade de medicamentos prescritos nessa unidade, sendo que as complicações relacionadas ao uso de medicações são o tipo de evento adverso mais comum na internação, representando 3 a $5 \%$ das reações adversas a medicamentos, que podem ser prevenidas em hospitais (HAMMES et al., 2008).

Das prescrições analisadas, 32,8\% apresentaram pelo menos uma interação medicamentosa contraindicada ou de gravidade maior considerando-se as evidências científicas boas ou excelentes. Diante desses resultados, nota-se que a necessidade identificar estas interações entre os medicamentos é de extrema relevância no ponto de vista de segurança do paciente, uma vez que já há estudos, também realizados em hospitais de ensino, onde as interações medicamentosas encontradas classificadas como contraindicadas ou de gravidade importante variaram de 41 a 51\%, sendo estas associadas a riscos 
de vida ou a intervenções para que se possam reduzir as possíveis reações adversas e os impactos negativos gerados, tanto no aspecto econômico, quando clínico (ALVIM et al., 2015; OLIVEIRA-PAULA et al., 2014). Outro estudo, realizado na UTI neonatal de um hospital escola do Estado de Mato Grosso, analisou 119 prescrições e $51,26 \%$ delas apresentaram interações medicamentosas (QUEIROZ et al., 2014).

Conforme autores, as interações medicamentosas nem sempre são controladas somente com a suspensão da combinação, mas também podem ser controladas com o ajuste de doses e o monitoramento de possíveis efeitos adversos, fazendo uma avaliação individual de riscos e benefícios (HAMMES et al., 2008). Dentre as prescrições dos pacientes envolvidos no estudo, observou-se um predomínio do sexo masculino $(60,6 \%)$, fato este que corrobora com estudo realizado em um hospital público do estado da Bahia, onde 64,0\% dos pacientes analisados eram do sexo masculino (PIEDADE et al., 2015).

Contudo, em outro estudo realizado em hospital particular de São Paulo 56,1\% dos pacientes era do sexo feminino, fato este que pode ser explicado devido a questões culturais, onde apesar de as mulheres buscarem mais pelos serviços de saúde, deve-se considerar que o envelhecimento apresenta formas distintas entre os sexos, e entende-se que as mulheres passam por uma maior debilitação biológica antes da morte, apresentando dessa forma uma chance maior de sofrer com doenças crônicas que os homens (VARELLO; COSTA; MASTROIANNI, 2013; QUEIROZ et al., 2015).

A idade média dos pacientes foi de 61,9 anos (18 a 89 anos), mostrando-se superior ao encontrado em outro estudo (PIEDADE et al., 2015), onde a média de idade dos pacientes analisado foi de 47,4 (18 a 86). Em perfil traçado de pacientes internados em uma UTI adulto de um hospital universitário a média de idade dos pacientes internados foi de 64,8 anos, indicando ainda que $49 \%$ dos pacientes analisados apresentavam idade superior a 60 anos (FAVARIN; CAMPONIGARAM, 2012).

No presente estudo, $64,8 \%$ dos pacientes analisados eram idosos, ou seja, possuíam idade igual ou superior a 60 anos. Pessoas com idade superior a 60 anos são mais predispostas à ocorrência de interações medicamentosas, visto que esses indivíduos em sua maioria são portadores de doenças crônicas, o que acarreta necessidade de associação de diversos medicamentos (ARAUJO et al., 2013). Quando hospitalizados, os pacientes acabam fazendo uso de polifarmácia, chegando a receber uma média de 7 fármacos por dia, sendo mais importante tal situação nos procedimentos em UTI que apresentam pacientes em modalidade crítica, utilizando diariamente grande número de medicamentos, chegando a alcançar uma média de até 15 fármacos.

Portanto esses indivíduos apresentam maior risco de desenvolver interações que em outras unidades, já que além do problema dos múltiplos medicamentos, há a complicação conferida pela gravidade da doença e da e pela eventual falência de órgãos (LIMA; CASSIANI, 2009). Esta média citada, está próxima a encontrada no estudo, quando os pacientes analisados tiveram uma média de 13,3 medicamentos prescritos no dia, sendo que o máximo de medicamentos prescritos por pacientes nas prescrições diárias foram de 21 e o mínimo de 6 medicamentos. Média inferior foi encontrada em outro estudo, onde os pacientes acompanhados apresentaram uma média de 6,8 medicamentos por prescrição médica, variando de um a 17 fármacos (PIEDADE et al., 2015).

O elevado número de medicamentos prescritos para pacientes internados em UTI indica um aumento de risco, onde o número de medicamentos é diretamente proporcional ao desenvolvimento de interações medicamentosas e efeitos adversos, aumentando o tempo de internação (CEDRAZ; SANTOS, 2014).

A interação mais frequente encontrada neste estudo, foi entre o medicamento ácido acetilsalićlico e enoxaparina (10,4\%), que quando administrados concomitantemente, podem causar o risco de eventos hemorrágicos, e a monitorização do paciente e de seus sinais e sintomas é o manejo clínico a ser seguido conforme a base de dados utilizada. O ácido acetilsalicílico é um salicilato com ação anti-inflamatório não esteroidal que inibe a agregação plaquetária com prolongamento do tempo de sangramento. As plaquetas perdem a capacidade de se agregar e formar trombos pelo resto de seu tempo de vida (7 a 10 dias). É utilizado normalmente na UTI devido a sua ação antiagregante plaquetária e interage com a enoxaparina interferindo na função plaquetária, podendo aumentar o risco de sangramento (KATZUNG, 2007). 
Já a morfina foi o medicamento mais envolvido em interação entre medicamento, estando presente em 34 dos casos nesse estudo. Este é um medicamento classificado como analgésico e sedativo muito utilizado no alivio a resposta ao estresse e diminuir as complicações em pacientes críticos internados em UTI (FERREIRA; HIDALGO; CAUMO, 2004). Sabe-se da necessidade de prescrições de analgésicos opióides, sendo a morfina um desses, para os pacientes idosos que estão internados e, com o aumento da hospitalização desta população se tem um potencial elevado para a ocorrência de interações entre os medicamentos, uma vez que os idosos fazem uso de polifarmácia (RIBEIRO; COSTA, 2015).

No hospital do estudo, a implementação das possíveis interações medicamentosas no sistema informatizado poderá contribuir para diminuir as tais interações, servindo como um alerta ao prescritor e a equipe, sugestão essa que será posteriormente discutida na Comissão de Segurança do Paciente da instituição em estudo, já que uma crescente atenção tem sido direcionada para as interações medicamentosas, nos últimos anos, principalmente, no meio hospitalar. Muitos programas informatizados têm sido desenvolvidos e apontados na literatura como importante ferramenta na revisão de prescrições médicas. Estes, quando utilizados em hospitais, têm demonstrado resultados satisfatórios, visto que se mostram capazes de reduzir as interações medicamentosas. Alguns autores relatam, como vantagens, agilidade na análise das prescrições, redução de erro de medicação, tempo de internação e gastos (QUEIROZ et al., 2014).

A média de dias que os pacientes permaneceram internados na UTI foi de 7 dias, tempo esse inferior ao relatado em outros estudos, onde o tempo médio de internação na UTI variou de 14,8 a 19,4 dias (OLIVEIRAPAULA et al., 2014; CARVALHO et al., 2013).

\section{CONCLUSÃO}

A identificação das interações medicamentosas possibilita evitar situações de insucesso terapêutico ou minimização do aparecimento de toxicidade medicamentosa pelo ajuste da posologia ou pelo uso de fármacos alternativos. O estudo identificou que em 32,8\% das prescrições apresentaram pelo menos uma potencial interação medicamentosa, o que enaltece a importância do trabalho de uma equipe clínica multiprofissional com a participação do farmacêutico clínico, já que as intervenções farmacêuticas podem contribuir para a diminuição dos eventos adversos evitáveis. Sendo assim, o presente estudo colaborou de forma significativa para o conhecimento das interações entre os medicamentos na UTI do hospital em estudo, mostrando que esta prática é importante para planejar as ações de melhoria e, assim, garantir a segurança do paciente em terapia intensiva.

\section{REFERÊNCIAS}

ALMEIDA, S.M.; AKAMINE, N. Prevalência e classificação de interações entre medicamentos dispensados para pacientes em terapia intensiva. Einstein (São Paulo), v. 5, n. 4, p. 347-351, 2007.

ALVIM, M.M.; SILVA, L.A.; LEITE, I.C.; SILVÉRIO, M.S. Eventos adversos por interações medicamentosas potenciais em unidade de terapia intensiva de um hospital de ensino. Rev Bras Ter Intensiva, v. 27, n. 4, p. 353-359, 2015.

ARAUJO, T.G.; RIEDER, M.M.; KUTCHAK, F.M.; FRANCO FILHO, J.W. Readmissões e óbitos após a alta da UTI: um desafio da terapia intensiva. Rev Bras Ter Intensiva, v. 25, n.1, p.32-38, 2013.

BRASIL. Ministério da Saúde. Secretária de Ciência, Departamento de Assistência Farmacêutica e Insumos Estratégicos. Formulário terapêutico nacional 2010. Brasília: [s.n.], 2010.

CARVALHO, R. E. F. L.; REIS, A. M. M.; FARIA, L. M. P.; ZAGO, K. S. A.; CASSIANI, S. H. B. Prevalência de interações medicamentosas em unidades de terapia intensiva no Brasil. Acta Paul Enferm, v. 26, n. 2, p. 150$157,2013$.

CEDRAZ, K. N.; SANTOS, M. C. J. Identificação e caracterização de interações medicamentosas em prescrições médicas da unidade de terapia intensiva de 
um hospital público da cidade de Feira de Santana, BA. Rev Soc Bras Clin Med, v. 12, n. 2, p. 1-7, 2014.

DOURADO ARRAIS, P. S.; BARRETO, M. L.; COELHO, H. L. L. Aspectos dos processos de prescrição e dispensação de medicamentos na percepção do paciente: estudo de base populacional em Fortaleza, Ceará, Brasil. Cad Saúde Pública, v.23, n.4, p.927-37, 2007.

FAVARIN, S.S.; CAMPONIGARAM, S. Perfil dos pacientes internados na unidade de terapia intensiva adulto de um hospital universitário. Rev Enferm UFSM, v. 2, n. 2, p. 320-329, 2012.

FERREIRA, M. B. C.; HIDALGO, M. P. L.; CAUMO, W. Analgésicos Opióides. In: FUCHS, F. D; WANNMACHER, L.; FERREIRA, M. B. C. Farmacologia clínica: fundamentos da terapêutica racional. 3. ed. Rio de Janeiro: Guanabara Koogan, 2004. p. 236-58.

GONÇALVES, S. S.; RODRIGUES, H. M. S.; JESUS, I. S.; CARNEIRO, J. A. O.; LEMOS, G. S. Ocorrência clínica de interações medicamentosas em prescrições de pacientes com suspeita de reação adversa internados em um hospital no interior da Bahia. Rev. Aten. Saúde, v. 14, n. 48, p. 32-33, 2016.

HAMMES, J. A.; PFUETZENREITER, F.; SILVEIRA, F; KOENIG, A.; WESTPHAL, G. A. Prevalência de potenciais interações medicamentosas droga-droga em unidade de terapia intensiva. Rev Bras Ter Intensiva, v.20, n.4, p.349-353, 2008.

KATZUNG, B. G. Farmacologia básica e clínica.10. ed. Rio de Janeiro: Guanabara Koogan, 2007.

LIMA, R. E. F.; CASSIANI, S. de B.Interações medicamentosas potenciais em pacientes de unidade de terapia intensiva de um hospital universitário. Rev Lat Am Enfermagem, v.17, n.2, 2009.

MICROMEDEX $^{\circledR}$. Healthcare Series. Greenwood Village, Colo: Thomson Reuters (Healthcare) Inc. Updated periodically.

OGA, S. Interações Medicamentosas. In: STORPIRTIS, S.; MORI, A. L. P. M.; YOCHIY, A.; RIBEIRO, E.; PORTA,
V. Farmácia clínica e atenção farmacêutica. Rio de Janeiro: Guanabara Koogan, p.78-85, 2008.

OLIVEIRA-PAULA, G. H.; PEREIRA, F.; PACCOLA, M. T.; MARTINS-JUNIOR, A. C.; DALLA-COSTA, E. M. O. Interações medicamentosas potencias em unidades de terapia intensiva de um hospital do Sul do Brasil. Semina, Londrina, v.35, n. 2, p. 21-30, 2014.

PAPADOPOULOS, J.; SMITHBURGER, P. L. Common drug interactions leading to adverse drug events in the intensive care unit: management and pharmacokinetic considerations. Crit Care Med, v.38, n.6, p.126-135, 2010.

PIEDADE, D.V.; SILVA, L. A. F.; VALASQUES, G. L.; LEMOS, L. B. Interações medicamentosas envolvendo antimicrobianos. Medicina, Ribeirão Preto, v. 48, n. 3, p. 295-307, 2015.

QUEIROZ, D. B.; OLIVEIRA, L. C.; ARAÚJO, C. M.; REIS, L. A. Perfil de interações de idosos de uma clínica de neurociências de um hospital público. Rev Enferm Contemporânea, v. 5, n. 1, p. 16-24, 2015.

QUEIROZ, K. C. B.; NASCIMENTO, M. F. S.; FERNANDES, V.; MIOTTO, F. A. Análise das Interações Medicamentosas Identificadas da UTI Neonatal da ICU-HGU. UNOPAR Cient Ciênc Biol Saúde, v.16, n.3, p. 203-207, 2014.

RIBEIRO, H. S. S.; COSTA, J. M. Acompanhamento farmacoterapêutico de idosos em uso de analgésicos opioides em um hospital de ensino. Rev. Bras. Farm. Hosp.,v. 6 n. 1, p. 18-23, 2015.

RODRIGUES, M. C. S.; OLIVEIRA, L. C. Erros na administração de antibióticos em unidade de terapia intensiva de hospital de ensino. Rev Eletr Enferm, v.12, n.3, p. 511-519, 2010.

SECOLI, S. R. Polifarmácia: interações e reações adversas no uso de medicamentos por idosos. Rev Bras Enferm., v. 63, n.1, p.136-140, 2010

SMITHBURGER, P. L.; KANE-GILL, S. L.; SEYBERT, A. L. Drug-drug interactions in cardiac and cardiothoracic intensive care units: an analysis of patients in an academic medical centre in the US. Drug Saf, v.33, n.10, p. 879$888,2010$.

SPRIET, I.; MEERSSEMAN, W.; DE HOON, J.; VON WINCKELMANN, S.; WILMER, A.; WILLEMS, L. Mini- 
series: II. Clinical aspects. Clinically relevant CYP450mediated drug interactions in the ICU. Intensive Care Med, v.35, n.4, p.603-612, 2009.

VARELLO, F. R.; COSTA, M. A.; MASTROIANNI, P. C. Potenciais interações medicamentosas responsáveis por internações hospitalares. Rev. Ciênc Farm Básica Apl., v. 34, p. 79-85, 2013.

Recebido em: 06 de outubro de 2016 Aceito em: 15 de fevereiro de 2017 